\title{
Towards 5D Optical Data Storage with High Writing Speed
}

\author{
Huijun Wang, Yuhao Lei, Xin Chang, Chun Deng, Gholamreza Shayeganrad, and Peter G. Kazansky \\ Optoelectronics Research Centre, University of Southampton, Southampton, SO17 1BJ, United Kingdom \\ Huijun.Wang@soton.ac.uk
}

Due to the information explosion in the 21 st century, data storage technologies to meet the growing demand for information preservation have attracted considerable interest. Based on self-assembled nanogratings produced by ultrafast laser writing in silica glass, recording and retrieval of multiplexed digital data with a nearly unlimited lifetime was demonstrated [1]. Three spatial dimensions and two optical ones (the slow axis orientation and the retardance) were exploited to realize 5D data storage. However, the drawbacks of low optical transmission in the visible range and low writing speed hinder its utility in practical applications. Recently, a new type of ultrafast laser-induced modification in silica glass, which consists of randomly distributed nanopores, was demonstrated, providing controllable birefringent structures with transmittance as high as $99 \%$ in the visible range [2,3]. 5D optical data storage with ultrahigh readout accuracy of multilayer data, up to $100 \%$ was achieved [4], but with the drawback of low writing speed of $0.2 \mathrm{kB} / \mathrm{s}$. Here, we report nanopores-based 5D data storage with an increased data writing speed up to $8 \mathrm{kB} / \mathrm{s}$ and higher data capacity by tighter focusing in combination with higher repetition rate, maintaining ultrahigh readout accuracy.

Data recording experiments were carried out with a $\mathrm{Yb}: \mathrm{KGW}$ mode-locked regenerative amplified femtosecond laser system (PHAROS, Light Conversion Ltd.) operating at $1030 \mathrm{~nm}$ with a repetition rate of $1 \mathrm{MHz}$ and a pulse duration of $300 \mathrm{fs}$. The laser beam was focused via a $0.31 \mathrm{NA}$ aspheric lens into silica glass sample mounted on a three-axial air-bearing translation stage (ABL-10150, Aerotech Ltd.)

(a)

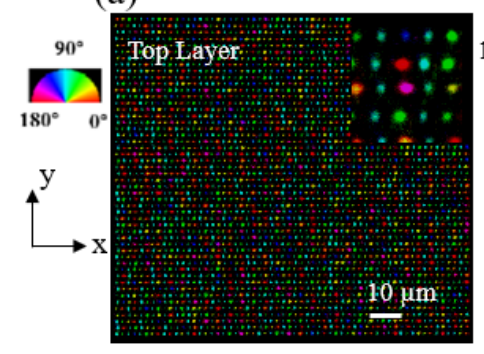

(b)

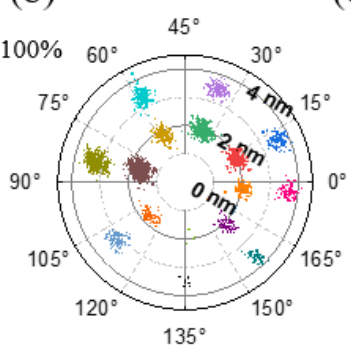

(c)

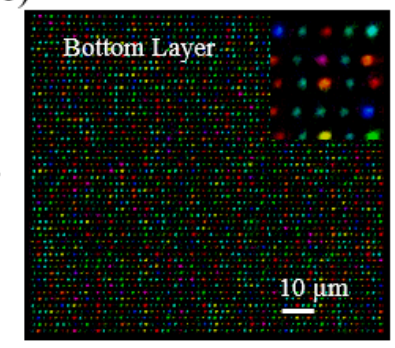

(d)

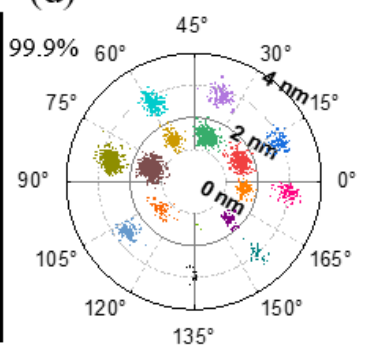

Fig. $15 \mathrm{D}$ data storage with high writing speed, demonstrated by the writing of 'The Hitchhiker's Guide to the Galaxy'. The energy per pulse was $190 \mathrm{~nJ}$. (a) and (c) The voxel images taken by the Abrio birefringence imaging system. Insets are enlargements of small region $10 \mu \mathrm{m} \times 10 \mu \mathrm{m}$. (b) and (d) Polar diagram of the measured retardance (2 levels) and azimuth (8 levels) of all voxels in (a) and (c).

5D data storage based on type $\mathrm{X}$ modification with a high recording rate was successfully demonstrated by writing the book of The Hitchhiker's Guide to the Galaxy in 42 plus 3 extra layers. For data recording, the azimuth was divided into 8 levels and the pulse number of 20 and 30 were used to achieve two distinguishable retardance levels, resulting in 4 bits ( $2 \times 8)$ of information per voxel [Fig. 1(a) and (c)]. The lateral voxel separation and layer separation were decreased to $2 \mu \mathrm{m}$ and $17.5 \mu \mathrm{m}$, respectively, by using a 0.3 NA objective, corresponding to the writing speed of $8 \mathrm{kB} / \mathrm{s}$ with the scanning speed of $30 \mathrm{~mm} / \mathrm{s}$. The retrieved accuracy of the top and the bottom layers was $100 \%$ and $99.9 \%$, respectively [Fig. 1(b) and (d)].

It is possible to increase the writing speed up to $\sim 40 \mathrm{kB} / \mathrm{s}$ and data capacity of $1.4 \mathrm{~TB} / \mathrm{disc}$ by further reducing the voxel separation and pulse number. Although the writing speed of $230 \mathrm{kB} / \mathrm{s}$ can be achieved with potential data capacity of $500 \mathrm{~TB} /$ disc by using pulse energy modulation method [5], additional error correction is necessary to achieve $100 \%$ data readout accuracy.

\section{References}

[1] J. Zhang et al., "Seemingly unlimited lifetime data storage in nanostructured glass," Phys. Rev. Lett. 112, 033901 (2014).

[2] R. Drevinskas et al., "Nanostructured optical element, method for fabrication and uses thereof," (2019).

[3] M. Sakakura et al., "Ultralow-loss geometric phase and polarization shaping by ultrafast laser writing in silica glass," Light, science \& applications, 915 (2020).

[4] H. Wang et al., "5D optical data storage with 100\% readout accuracy in silica glass," CLEO conference, USA, 11-13 May 2021 (submitted).

[5] Y. Lei et al., "Towards the smallest anisotropic structures by ultrafast laser writing in silica glass," CLEO: Science and Innovations, DC United States, (2020) 